\title{
The association between maternal-reported responses to infant crying at 4 weeks and 6 months and offspring depression at 18: a longitudinal study
}

\author{
Catherine J. Williams ${ }^{1}$ • David Kessler ${ }^{2}$. \\ Charles Fernyhough $^{4} \cdot$ Glyn Lewis $^{3} \cdot$ Rebecca M. Pearson ${ }^{2}$
}

Received: 19 March 2015 / Accepted: 7 December 2015 / Published online: 2 February 2016

(C) The Author(s) 2016. This article is published with open access at Springerlink.com

\begin{abstract}
The purpose of the present study is to examine the association between maternal response to infant crying and the psychological health of the child in later life. Using data from the Avon Longitudinal Study of Parents and Children (ALSPAC) cohort, consisting of 15,247 pregnancies, 10,278 with exposure variables and 3201 complete cases were identified as having exposure, covariate and outcome data. Using a postal questionnaire, mothers were asked regarding their infant at 4 weeks and 6 months, 'If they cry what do you do?': (a) pick them up immediately; (b) if they cry, leave them for a while, and if they do not stop, pick them up; or (c) never pick them up until you are ready. Outcome was an International Statistical Classification - 10th revision criteria (ICD-10) diagnosis of depression at 18 years for the infant. Offspring of mothers who at 4 weeks reported that they never picked their infants up until they were ready were more likely to have depression at 18 years $(\mathrm{OR}=2.06, \mathrm{CI} 0.95-4.47$, adjusted for sociodemographic confounding variables). There was no evidence for an association at 6 months. Including adjustment variables reduced the strength of our association; an observed objective measure of maternal response rather than a selfreport may have more accurately determined the mother's
\end{abstract}

Catherine J. Williams

epzcjw@bristol.ac.uk

1 Centre for Academic Primary Care, Canynge Hall, 39 Whatley Road, Bristol BS8 2PS, UK

2 Centre for Mental Health, Addiction and Suicide Research, Oakfield House, Oakfield Road, Bristol BS8 2BN, UK

3 Division of Psychiatry, Charles Bell House, 67-73 Riding House St, London W1W 7EJ, UK

4 Department of Psychology, Durham University, Science Site, South Road, Durham 1 DH1 3LE, UK actual responses. There is some evidence for an association between maternal reporting of responses to infant crying at 4 weeks and risk of developing depression at 18 years. If this association is found to be causal, interventions encouraging mothers to represent and respond to their infants' emotional states may help prevent offspring depression.

Keywords Infant crying - Adolescent depression .

Mind-mindedness · Attachment · Avon Longitudinal Study of Parents and Children · Emotional regulation

\section{Introduction}

Depression in adolescence is an important cause of morbidity with a prevalence of $4 \%$ at 15 years and $16 \%$ at 18 years (Jaffee et al. 2002). In one US study, $45 \%$ of those who had been depressed aged 16 years experienced a recurrence before the age of 23 years and further episodes were easier to provoke (Lewinsohn et al. 1999a, b).

Depression can be viewed as a disorder of poor emotion regulation (Jorman and Gotlib 2010). The development of emotional regulation starts in infancy in the context of interactions with primary caregivers (McElwain and BoothLaForce 2006; Bigelow et al. 2010). In infancy, mother and infant emotion regulation are interdependent and form a basis from which the child learns self-regulation (Bornstein 2014; Choe et al. 2013). Through sensitive observation and responses, mothers can facilitate the infant's reactions to internal and external stimuli, and this in turn may help the infant achieve a well-regulated emotional state (Thompson 1994). Maternal support at times of distress is particularly important in facilitating emotional regulation and with the development of a secure child-mother attachment (McElwain and BoothLaForce 2006; Bigelow et al. 2010) because it allows the 
infant to develop a sense of security that their needs will be met and provides a model on which they can learn to selfsoothe. We therefore hypothesise that maternal response to infant distress is important to the development of emotion regulation. Given the importance of emotion regulation for depression, we hypothesise that such maternal responses will in turn be associated with a long-term risk of depression. Understanding this relationship could provide opportunities for early interventions for mothers and infants at risk.

Although many studies exist linking parenting behaviour to emotional regulation in later childhood (McElwain and Booth-LaForce 2006; Meins et al. 2001; Murray et al. 2011; Halligan et al. 2007), previously, there have been no large longitudinal studies looking at offspring-reported outcomes in early adulthood in relation to maternal response in infancy. The Avon Longitudinal Study of Parents and Children is a large representative birth cohort with available data stretching from the antenatal period until early adulthood. We used the cohort data to investigate the association between selfreported maternal response to crying early in the child's life (4 weeks and 6 months of age) and the prevalence of adolescent depression in offspring using a validated International Statistical Classification-10th revision criteria (ICD-10) diagnosis of depression - the computerised revised clinical interview schedule (CIS-R) (Lewis et al. 1992).

\section{Methods}

\section{Sample}

The sample comprised participants from the Avon Longitudinal Study of Parents and Children (ALSPAC). All pregnant women resident in the former Avon Health Authority in South-West England with an estimated delivery date between 1 April 1991 and 31 December 1992 were invited to take part, resulting in a core cohort of 14,541 pregnancies. 14, 062 were live births and 13,988 babies were alive at 12 months of age (Boyd et al. 2013). This resulted in a total cohort of 15,247 pregnancies, 14,775 live births and 14, 701 babies alive at 12 months of age (Boyd et al. 2013). Comparison with the 1991 National Census data confirmed that the sample was broadly representative of the general population (Boyd et al. 2013).

The samples, however, had some differences to the general population and from the local Avon population by having a greater proportion of mothers who were married, were owneroccupiers, who had a car in the household and had a higher socioeconomic position (Fraser et al. 2013). Non-White minority ethnic groups were underrepresented (Boyd et al. 2013). By 16 years, offspring tended to have achieved higher educationally when compared to national Key Stage 4 samples and those who were lost to follow-up tended to have lower educational attainment (Fraser et al. 2013; Boyd et al. 2013). Ethical approval was obtained from the ALSPAC Law and Ethical Committee and the Local Research Ethics Committees (see http://www.alspac.bris.ac.uk).

Detailed information including 59 questionnaires has been collected since the cohort started (Boyd et al. 2013) (please note that the study website contains details of all the data that is available through a fully searchable data dictionary available at http://www.bristol.ac.uk/alspac/researchers/dataaccess/data-dictionary/).

\section{Exposure variables}

We identified questions on how mothers responded to their crying babies from more extensive questionnaires on child and carer behaviour in infancy. The question selected was part of a maternal self-report questionnaire sent out at 4 weeks and 6 months. It was chosen for analysis as it was posed at two time points and was a representation of what the mother actually did rather than what she thought should be done. Mothers were asked how they responded to their infant crying: If they cry, what do you do?

1. Pick them up immediately.

2. Leave them for a while, and if they do not stop, pick them up.

3. Never pick them up until you are ready.

4. Varies.

Answer 4 was dropped from the questionnaire as it did not identify the mother's usual pattern of behaviour (number $=503$ ). Answers 1 and 2 were deemed more child-centred maternal reports of responses to distress. Answer 3 was regarded as a marker of a more mother-centred rather than child-centred response to distress and therefore represented a lower or nonattuned response (Meins et al. 2011) and a mother who was less likely to pick up the infant. It is considered to be child-centred because the response is conditional on a cue/need from the infant, if they do not stop crying. In contrast, the response 'pick them up when you are ready' is conditional on the needs of the parent. Answer 1 is coded from here on as 'pick them up immediately', answer 2 'leave for a while then pick them up' and answer 3 'pick them up when ready'.

\section{Outcome variable}

Outcome was an ICD-10 diagnosis of depression at 18 using the computerised CIS-R (Lewis et al. 1992). This is based on a diagnosis of depression using the International Classification of Diseases, 10th revision criteria. The interview is fully standardised, and results are reliable whether conducted by a clinically trained interviewer or self-administered on the computerised version (Lewis et al 1992; Patton et al. 1999; 
Bell et al. 2005). The outcome measure is a binary variable indicating the presence of absence of a primary diagnosis of major depression on the CIS-R.

\section{Statistical analysis}

The association was analysed using logistic regression in STATA version 12 . The following potential confounders were investigated: antenatal depression at 32-week gestation (Edinburgh Postnatal Depression (EPDS) score, Cox and Holden 1994), postnatal depression at 8 weeks (Edinburgh Depression Score), maternal education at 32-week gestation (CSE or below, A level, degree), breastfeeding at 6 months (never, stopped, still feeding), gender (male/female), number of siblings at 6 months, marital conflict at 8 months (Rutter and Quinton 1984), homeowner at 8 months (yes/no), type of dwelling at 8 months (detached, semi-detached, terraced house, flat/maisonette, rooms, other), neighbourhood at 8 months (very good/good/not very good), social class (1-6), maternal age at time of delivery, multiple pregnancy at time of delivery and single-parent status at 8 months (single/partner).

\section{Selection of adjustment variables}

Final adjustment variables were as follows: antenatal depression, maternal education, breastfeeding, gender, number of siblings, marital conflict and maternal age. Homeowner, type of dwelling and neighbourhood were not used in the final analysis because maternal education is highly correlated with all of these variables and provides a good proxy measure for socioeconomic status which was measured early in the study and is relatively complete. Including them would lead to a diminished sample size reducing statistical power and potentially introducing bias unnecessarily. Postnatal depression was not included as it is correlated strongly with antenatal depression measured only a few weeks previously $(r=0.6)$. Furthermore, the available measure of postnatal depression was taken after the exposure of response to crying at 4 weeks and therefore may be a consequence of the response to crying. This would be on the causal pathway. Therefore, ongoing maternal depression would be considered as a mediating pathway variable and full investigation of this would be beyond the scope of the current paper. The antenatal depression measure therefore provides the most appropriate available variable to account for the important potential confounding effect of maternal depression. Social class was dropped because the large amount of missing data reduced power and again similar sociodemographic variables were already being included. Single parent could not be used in our imputation model for missing values, as it was too rare an event to impute, so it was dropped from our complete-case analysis also for consistency. There was no evidence that this variable influenced response to crying. Twins as a paired event were accounted for using robust estimation of standard errors and were not imputed, as there was no missing data for twins. All available data and complete cases were analysed with and without adjustment variables. In total, 10,278 mother-infant dyads had both of the exposure variables and 3201 were complete cases across all exposure, adjustment variables and outcome data.

\section{Analysis of missing values}

Missing values were processed using multiple imputations by chained equations (MICE) on STATA version 12 (White et al. 2011). Ignoring missing data can introduce bias by assuming that data are missing completely at random. The ALSPAC cohort has many sociodemographic variables that can be used to predict missing information based on the observed data. All of our final adjustment variables were used for imputation plus other adolescent measures of offspring depression and sociodemographic variables to predict missing data across 80 imputed datasets (full list of auxiliary variables available on request). Monte Carlo errors were less than $1 \%$ of the standard error and FMI values were no larger than 0.03 . We were able to impute up to a starting sample of 7266 for whom there was complete exposure data from the mother and at least one earlier measure of depression in the child.

Analysis was repeated on these datasets, combining estimates from each of the 80 imputed datasets using Rubin's rules (White et al. 2011).

\section{Results}

\section{Maternal characteristics and change in maternal response between 4 weeks and 6 months}

Table 1 shows the characteristics of the mothers at 4 weeks by their response to infant crying. The largest overall group, 'leave for a while then pick them up', was chosen as the reference group. There are a few differences between the groups: The 'pick them up immediately' group tended to be from a higher social class and was more likely to still be breastfeeding and has obtained a higher educational status. Mothers who responded by saying 'pick them up when ready' were less likely to be homeowners than in the other groups but were otherwise similar in characteristics to the reference group. Mothers who 'leave for a while then pick them up' were more likely to be from a lower social class.

In the sample as a whole, $75 \%$ of mothers gave the same response at 4 weeks and 6 months (kappa 0.43 ). The proportion of those who changed their response was greatest in the group of interest, the 'pick them up when ready' group. At 4 weeks, there were 79 mothers in this group, and at 6 months, it had increased in size to 123 mothers. Only 27 (34\%) of those who gave this response at 4 weeks remained in this group at 6 months. 
Table 1 Characteristics of mothers according to response to crying at 4-week core ALSPAC sample, $n=10,278$

\begin{tabular}{|c|c|c|c|c|c|c|}
\hline Maternal characteristic & Total number & Scale & $\begin{array}{l}\text { Leave for a while } \\
\text { then pick them up }\end{array}$ & $\begin{array}{l}\text { Pick them up } \\
\text { immediately }\end{array}$ & $\begin{array}{l}\text { Pick them up } \\
\text { when ready }\end{array}$ & $\begin{array}{l}\text { Chi-square } \\
\text { and } p \text { values }\end{array}$ \\
\hline Antenatal depression & 10,643 & $\begin{array}{l}\text { Mean (SD) Edinburgh } \\
\text { Depression scale }\end{array}$ & $\begin{array}{l}7.1(\text { SD } 5.1) \\
n=7484\end{array}$ & $\begin{array}{l}6.7(\text { SD 5) } \\
n=2932\end{array}$ & $\begin{array}{l}7.3(\text { SD } 5.2) \\
n=227\end{array}$ & $p=0.001$ \\
\hline Postnatal dep. 8 weeks & 10,672 & $\begin{array}{l}\text { Mean (SD) Edinburgh } \\
\text { Depression scale }\end{array}$ & $\begin{array}{l}6.1(\mathrm{SD} 4.8) \\
n=7455\end{array}$ & $\begin{array}{l}5.9(\mathrm{SD} 4.8) \\
n=2986\end{array}$ & $\begin{array}{l}6.1(\mathrm{SD} 4.7) \\
n=231\end{array}$ & $p=0.13$ \\
\hline Maternal degree & 10,342 & $\begin{array}{l}\text { CSE or below } \\
\text { A level } \\
\text { Degree }\end{array}$ & $\begin{array}{l}n=4777 \\
n=1666 \\
n=772\end{array}$ & $\begin{array}{l}n=1513 \\
n=795 \\
n=600\end{array}$ & $\begin{array}{l}n=147 \\
n=38 \\
n=34\end{array}$ & Chi-square $=251 p=0.00$ \\
\hline Maternal social class & 9002 & $\begin{array}{l}\text { I-II } \\
\text { III-VI }\end{array}$ & $\begin{array}{l}n=2114 \\
n=4162\end{array}$ & $\begin{array}{l}n=1160 \\
n=1388\end{array}$ & $\begin{array}{l}n=65 \\
n=113\end{array}$ & Chi-square $=124 p=0.00$ \\
\hline Maternal age & 11,596 & Mean age years (SD) & $\begin{array}{l}28(\mathrm{SD} 4.8) \\
n=8131\end{array}$ & $\begin{array}{l}29(\mathrm{SD} 4.9) \\
n=3216\end{array}$ & $\begin{array}{l}28(\mathrm{SD} 4.8) \\
n=249\end{array}$ & $p=0.00$ \\
\hline Still breastfeeding & 10,280 & $\begin{array}{l}\text { Never } \\
\text { Stopped } \\
\text { Still feeding }\end{array}$ & $\begin{array}{l}n=1973 \\
n=3505 \\
n=1688\end{array}$ & $\begin{array}{l}n=475 \\
n=1251 \\
n=1170\end{array}$ & $\begin{array}{l}n=57 \\
n=102 \\
n=59\end{array}$ & Chi-square $=326 p=0.00$ \\
\hline No. of siblings & 11,596 & Mean (SD) & $\begin{array}{l}0.8(0.9) \\
n=8131\end{array}$ & $\begin{array}{l}0.8(1.0) \\
n=3216\end{array}$ & $\begin{array}{l}1.1(0.9) \\
n=249\end{array}$ & $p=0.58$ \\
\hline Female gender & 5606 & & $n=3907$ & $n=1570$ & $n=129$ & Chi-square $=1.77 p=0.41$ \\
\hline Marital conflict ${ }^{\mathrm{a}}$ & 9585 & Mean (SD) of scale & $\begin{array}{l}21.6(\text { SD } 7.2) \\
n=6699\end{array}$ & $\begin{array}{l}21.5(\text { SD } 7.3) \\
n=2686\end{array}$ & $\begin{array}{l}22.5(\text { SD } 7.3) \\
n=200\end{array}$ & $p=0.59$ \\
\hline Homeowner & 10,189 & Yes & $n=5546$ & $n=2263$ & $n=142$ & Chi-square $=22.58 p=0.00$ \\
\hline Neighbourhood & 10,233 & $\begin{array}{l}\text { Very good/good } \\
\text { Not very good }\end{array}$ & $\begin{array}{l}n=6752 \\
n=402\end{array}$ & $\begin{array}{l}n=2690 \\
n=173\end{array}$ & $\begin{array}{l}n=197 \\
n=19\end{array}$ & Chi-square $=5.59 p=0.23$ \\
\hline $\begin{array}{l}\text { Offspring ICD-10 } \\
\text { depression at } \\
18 \text { years }\end{array}$ & & & $n=2613$ & $n=1202$ & $n=79$ & \\
\hline
\end{tabular}

${ }^{\mathrm{a}}$ See Rutter and Quinton (1984)

In the sample as a whole, there is a tendency for mothers to move to a less responsive group over time from 'pick them up immediately' to one of the other two responses or from 'leave for a while then pick them up' to 'pick them up when ready'.

In order to investigate change in response patterns from 4 weeks to 6 months, we derived a four-level categorical variable with the following levels: (1) child-centred response at both 4 weeks and 6 months, (2) child-centred response at 4 weeks and then a shift to parent-centred response at 6 months, (3) parent-centred and then shift to child-centred response at 6 months and (4) parent-centred response at both time points.

If, as speculated, a shift from child-centred to parentcentred responsiveness is reflective of developmentally appropriate independence, then we would expect to see that offspring whose mothers show this pattern are not at risk, but those who show the reverse would be most at risk. Indeed, this is what we found.

\section{Association with depression at 18 years}

We found some evidence for an association between the development of depression at 18 years with the maternal response at 4 weeks - 'pick up when ready' - compared to the 'leave for a while then pick them up' maternal response, with an OR of 2.20 (CI 1.02-4.73, $p=0.04$ ). This is an increase from a prevalence of depression at 18 years of $7.04 \%$ in our baseline group-'leave for a while then pick them up'-to $13.92 \%$ in our 'pick up when ready' group. Complete cases with adjustment variables gave an OR of 2.06 (CI 0.95-4.49, $p=0.07)$. When taking into account the exposure at 6 months, there is still evidence for an association at 4 weeks with an OR of 2.32 (CI 1.03-5.22, $p=0.04$ ) (see Table 2, complete cases combined model with adjustment variables). No single adjustment variable was found to have a major confounding influence, but there was a small contribution from each leading to the overall reduction in OR. Results are shown for 4 weeks and 6 months in Tables 2 and 3. There was no evidence for an association between maternal responses at 6 months and depression (complete cases with adjustment variables OR 0.81 , CI 0.32-2.04, $p=0.65$; see Table 3). We have now included a secondary analysis looking at a continuous symptom score of depression derived from symptom-level questions on the CIS$\mathrm{R}$. We find a similar pattern of results with offspring of mothers who reported they never picked their infants up until they were ready at 4 weeks having higher depression symptom scores (regression coefficient 1.4 (95\% CI 0.4 to 2.3), 
$p=0.006)$ than those mothers who took a child-centred approach. Again, there was no association between maternal responses at 6 months (regression coefficient -0.4 (95\% CI -1.3 to 0.4$), p=0.296$ ).

As compared to being child-centred at both time points, offspring of mothers who showed a parent-centred response and then shift to child-centred response at 6 months were 2.5 times as likely to be depressed at 18 (95\% CI 1.1 to 3.3, $p=0.022$ ). In contrast, there was no evidence that offspring of mothers who show a more developmentally appropriate shift to child-centred response at 4 weeks and then a shift to parent-centred response at 6 months were not at risk (OR for depression at $18,0.88,95 \%$ CI 0.35 to $2.1, p=0.781$ ) (Fig. 1).

\section{Missing values}

We used multiple imputations using the STATA 12 command ice to account for missing values. Accounting for missingness at 4 weeks gave us an OR of 1.79, a CI of 0.94-3.42 and a $p$ value of 0.08 . With adjustment variables, this drops further to an OR of 1.66, a CI of 0.86-3.19 and a $p$ value of 0.13 (see Tables 2 and 3). Imputing up to only those with complete outcome gave similar results to complete-case analysis.

\section{Discussion}

We found some evidence to support the hypothesis that there is an association between the maternal-reported response 'pick up when ready' compared to the baseline maternal response 'leave for a while then pick them up' and later offspring depression at age 18. There was no evidence for an association between different maternal responses to crying in 6-month-old infants and depression at age 18 .

\section{Strengths and limitations}

Two key strengths of this study are the size of the cohort and the unique 18-year duration of follow-up with an ageappropriate diagnostic instrument for depression at 18 years. Although other longitudinal studies exist looking at early infant factors leading to adolescent depression, none have as large a cohort size or availability of adjustment variables as our study (Murray et al. 2011, Halligan et al. 2007). In addition, the cohort is broadly representative of the general population, making its findings more generalisable.

The overall effect was attenuated with the inclusion of adjustment variables and after accounting for missing values. There was no single adjustment variable that substantially diminished the association. After dealing with missing data, there was further weakening of this association. This could suggest an overestimation of the overall effect in the complete-case sample as compared to the imputed sample.

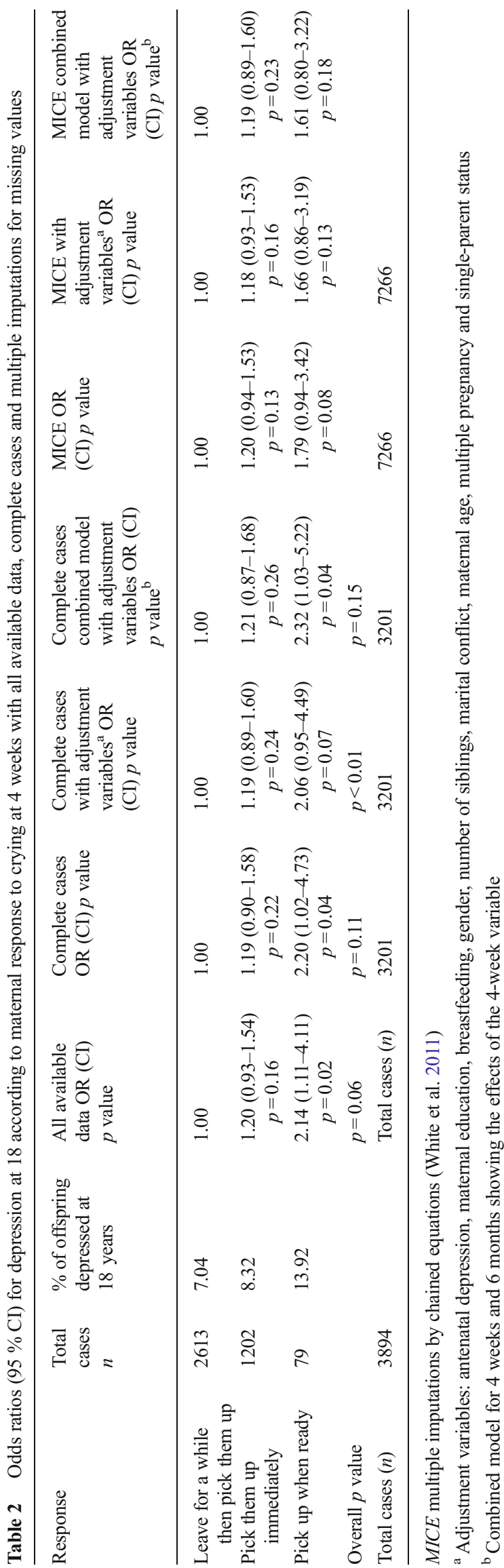


However, it is also likely that in both samples, the association was underestimated due to measurement error in both the outcome and exposure measures (i.e. underreporting of the less socially desirable parental responses). Therefore, our findings should be followed up in future studies.

Our question of interest addresses maternal-reported behavioural response to infant distress. It is unclear exactly how the maternal response on the items included relates to behavioural response to infant crying. For example, it is possible that mothers perceived 'pick up when ready' as a harsh response and many mothers who avoid picking up their infant when they cry did not report this answer. In addition, the responses only relate to picking up the infant, not to other types of comforting such as talking to the infant or offering a dummy. The questions may have been interpreted differently by different mothers and therefore might not represent the attitudes that we have ascribed to them. However, it seems reasonable to infer that a mother who agrees with the attitude 'pick up when ready' is less likely to behaviourally respond to an infant in distress. There is substantial evidence that attitudes towards parenting are associated with parenting behaviour (Grusec and Danyliuk 2014).

The findings should therefore stimulate further research into the role of maternal response towards infant distress for the development of offspring depression and other aspects of child development.

\section{Possible underlying mechanisms}

In the 'Introduction', we noted that infant emotion selfregulation (ability to understand and control emotions) develops in the context of interactions with primary caregivers. One possible interpretation of the present findings is that mothers with the response 'pick up when ready' are less likely to form a secure attachment (where the infant feels supported emotionally) with their infant and that this in turn increases the development of later vulnerability to depression (Allen et al. 2007; Milne and Lancaster 2001; Muris et al. 2001; Olsson

\section{Proportion of offspring with depression according to maternal responses to crying at 4 weeks and 6 months}

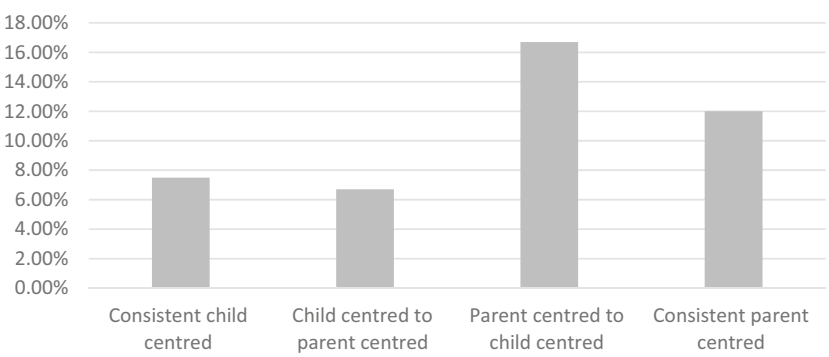

Fig. 1 Proportion of depression in offspring at 18 according to pattern of parental response in infancy 
et al. 1999; Salzman 1996). This would also be congruent with a growing body of evidence that several indices of childfocused parenting such as 'mind-mindedness' - the mothers' ability to 'tune in' to the offspring's mental state (including emotions) - and related concepts such as maternal sensitivity and 'appropriate and warm responses to infant cues (including emotions)', have long-term developmental effects (e.g. Meins et al. 2001; 2003, Murray et al. 2011).

\section{Reverse causality}

It is possible that more 'emotionally vulnerable' infants evoke a 'leaving to cry' response from their mothers, because these infants are more difficult to soothe. It is this emotional vulnerability that is then also associated with later depression in the child. However, if this is the case, we would expect to see an association between the 'pick up when ready' response at 6 months (when the mother has had more time to change her responses to the particular temperament of the child) as well as at 4 weeks. We also adjusted for prior maternal depression (during pregnancy) to control for the possibility that depressed mothers may respond differently to their babies and have offspring more vulnerable to later depression.

We still have to address the question of why no association between maternal response to crying and later depression was observed at 6 months. One possibility is that, by 6 months, a mother will have a better understanding of her infant's behaviour and will identify needs that require maternal support more readily. The secondary analysis looking at patterns of maternal response to crying from 4 weeks to 6 months is consistent with the hypotheses regarding the appropriateness of maternal response at different stages of development, because infants of mothers who show a transition from infant-centred to parentcentred response did not appear to be at later risk.

It may also be that each of the three maternal responses means something different to a mother with an infant of 4 weeks compared to an infant of 6 months. There may have been a change in perception or knowledge of how to respond to a crying infant based on peer group, family influences or health visitor advice. This may be especially true of our response of most interest 'pick up when ready'. It is likely that mothers view neonates as more vulnerable than older infants who have some selfregulatory capacity.

In support of this view, interventions for troublesome infant crying include leaving the infant to cry to give them the opportunity to self-soothe, briefly comforting the infant then allowing them to self-soothe or a parent staying in the room with the infant while they settle (Mumsnet 2012, Owens et al. 1999).

These interventions are used in infants over 6 months, and although evidence is limited, many websites and forums advocate their use (Hiscock and Wake 2002, Mumsnet 2012). The above behavioural-based interventions are generally not promoted in infants as young as 4 weeks (Douglas and Hill 2011).

Recommended interventions in this period are instead focused around mother-infant reciprocity, where the mother interacts with her infant according to his/her needs (for example breastfeeding on demand) and increased infant carrying (Douglas and Hill 2011; Hunziker and Barr 1986). At both 4 weeks and 6 months, we found no difference between the two child-centred responses 'pick them up immediately' and 'leave for a while then pick them up'. This may reassure mothers that leaving their child to settle briefly even at 4 weeks has no adverse effect on later depression.

Other practical implications of the present study include the possibility of developing family-based interventions encouraging mothers to respond appropriately to their crying infants. There are already precedents for such interventions in approaches based on increasing mind-mindedness in caregivers (e.g. Colonnesi et al. 2012).

\section{Conclusion}

There is some evidence to suggest that how a mother responds to her crying infant at 4 weeks may have an effect on the development of depression in her offspring at 18 years. If this finding is confirmed, a family-based intervention at 4 weeks encouraging mothers to respond appropriately to their crying infant has the potential to reduce rates of offspring depression at 18 . These results are potentially of importance, but further replication and evaluation of any interventions that are developed are needed. Future studies could use an observational measure or externally validate a self-reported questionnaire to reduce the risk of underreporting in our 'pick up when ready' group.

Acknowledgments We are grateful to all the families who took part, the midwives for help in recruiting them and the whole ALSPAC team, which includes interviewers, computer and laboratory technicians, clerical workers, research scientists, volunteers, managers, receptionists and nurses.

Funding body agreements and policies This study received funding from the UK Medical Research Council (grant ref: 74882), the Wellcome Trust (grant ref: 076467) and the University of Bristol which provided core support for ALSPAC. The current project was supported by Wellcome grants held by GL (grant ref: $084268 / \mathrm{Z} / 07 / \mathrm{Z}$ ) and CF (grant ref: 098455). This article is the work of the authors, and Dr. C J Williams will serve as guarantor for the contents of this article and the analyses of data.

Compliance with ethical standards Ethical approval for the study was obtained from the ALSPAC Ethics and Law Committee and the local research ethics committees. 
Conflict of interest $\mathrm{CW}$ was funded through an Academic Clinical Fellowship through the National School of Primary Care Research; staffing costs were met for GL, DK and RP through the University of Bristol and for CF through the University of Durham.

Open Access This article is distributed under the terms of the Creative Commons Attribution 4.0 International License (http:// creativecommons.org/licenses/by/4.0/), which permits unrestricted use, distribution, and reproduction in any medium, provided you give appropriate credit to the original author(s) and the source, provide a link to the Creative Commons license, and indicate if changes were made.

\section{References}

Allen JP, Porter M, McFarland C, Boykin K, Marsh P (2007) The relation of attachment security to adolescents' paternal and peer relationships, depression, and externalizing behaviour. Child Dev 78: 1222-1239

Bell T, Watson M, Sharp D, Lyons I, Lewis G (2005) Factors associated with being a false positive on the General Health Questionnaire. Soc Psychiatry Psychiatr Epidemiol 40(5):402-407

Bigelow A, Maclean K, Proctor J, Myatt T, Gillis R, Power M (2010) Maternal sensitivity throughout infancy: continuity and relation to attachment security. Infant Behav Dev 33(1):50-60

Bornstein MH (2014) Human infancy and the rest of the lifespan. Annu Rev Psychol. 65:121-158

Boyd A, Golding J, Macleod J, Lawlor DA, Fraser A, Henderson J, Molloy L, Ness A, Ring S, Davey Smith G (2013) Cohort profile: the children of the 90s'- the index offspring of the Avon Longitudinal Study of Parents and Children. Int J Epidemiol 42(1):111-127

Choe DE, Olson SL, Sameroff AJ (2013) Effects of early maternal distress and parenting on the development of children's self-regulation and externalizing behavior. Dev Psychopathol 25(2):437-453

Colonnesi C, Wissink IB, Noom MJ, Asscher JJ, Hoeve M, Stams GJJM, Polderman N, Kellaert-Knol MG (2012) Basic trust: an attachmentoriented intervention based on mind-mindedness in adoptive families. Res Social Work Prac 23(2):179-188

Cox J, Holden J (1994) Perinatal psychiatry: use and misuse of the Edinburgh Postnatal Depression Scale. Gaskell, London

Douglas PS, Hill PS (2011) The crying baby: what approach? Curr Opin Paediatr 23:523-529

Fraser A, MacDonald-Wallis C, Tilling K, Boyd A, Golding J, Davey Smith G, Henderson J, Macleod J, Molloy L, Ness A, Ring S, Nelson SM, Lawlor DA (2013) Cohort profile: the Avon Longitudinal Study of Parents and Children: ALSPAC mothers cohort. Int J Epidemiol 42(1):97-110

Grusec J, Danyliuk T (2014) Parents' attitudes and beliefs: their impact on children's development. Encyclopaedia on early childhood development., http://www.child-encyclopedia.com/parenting-skills/ according-experts/parents-attitudes-and-beliefs-their-impactchildrens-development. Accessed 25th Feb 2015

Halligan SL, Cooper PJ, Healy SJ, Murray L (2007) The attribution of hostile intent in mothers, fathers and their children. J Abn Child Psych 35(4):594-604

Hiscock H, Wake M (2002) Randomised controlled trial of behavioural infant sleep intervention to improve infant sleep and maternal mood. BMJ 324:1062
Hunziker U, Barr R (1986) Increased carrying reduces infant crying: a randomized controlled trial. Pediatrics 77:641-648

Jaffee S, Moffitt TE, Caspi A, Fombonne E, Poulton R, Martin J (2002) Differences in early childhood risk factors for juvenile-onset and adult onset depression. Arch Gen Psychiatry 58:215-222

Jorman J, Gotlib I (2010) Emotion regulation in depression: relation to cognitive inhibition. Cogn Emot 24(2):281-298

Lewinsohn PM, Rohde P, Klein DN, Seeley JR (1999a) Natural course of adolescent major depressive disorder: I. Continuity into young adulthood. J Am Acad Child Adolesc Psychiatry, 38 No,1, 56-63

Lewinsohn PM, Allen NB, Seeley JR, Gotlib IH (1999b) First onset versus recurrence of depression: differential processes of psychosocial risk. J Abnorm Psych 108:483-489

Lewis G, Pelosi AJ, Araya R, Dunn G (1992) Measuring psychiatric disorder in the community: a standardized assessment for use by lay interviewers. Psychol Med 22(No2):465-486

McElwain N, Booth-LaForce C (2006) Maternal sensitivity to infant distress and non distress as predictors of infant-mother attachment security. J Fam Psych 20(2):247-255

Meins E, Fernyhough C, Fradley E, Tuckey M (2001) Rethinking maternal sensitivity: mothers 'comments on infants' mental processes predict security of attachment at 12 months. J Child Psychol 42(5): 637-648

Meins E, Fernyhough C, Wainwright R, Clark-Carter D, Das Gupta M, Fradley E, Tuckey M (2003) Pathways to understanding mind: construct validity and predictive validity of maternal mind-mindedness. Child Dev 74:1194-1211

Meins E, Fernyhough C, Arnott B, Leekam S, Turner M (2011) Mother versus infant-centered correlates of maternal mind-mindedness in the first year of life. Infancy 16:137-165

Milne L, Lancaster S (2001) Predictors of depression in female adolescents. Adolescence 36(142):207-223

Muris P, Meesters C, Marion van Melick L (2001) Self-reported attachment style, attachment quality, and symptoms or anxiety and depression in young adolescents. Pers Individ Differences 30:809-818

Murray L, Artech A, Fearon P, Halligan S, Goodyer I, Cooper P (2011) Maternal postnatal depression and the development of depression in offspring up to 16 years of age. J Am Acad Child Adolesc Psychiatry 50(5):460-470

Olsson G, Nordstrom ML, Arinell H, Von Knorring AL (1999) Adolescent depression: social network and family climate- a case controlled study. J Child Psychol Psychiatry 40:227-237

Owens L, France KG, Wiggs L (1999) Behavioural and cognitivebehavioural interventions for sleep disorders in infants and children: a review. Sleep Med Rev 3(4):281-302

Patton GC, Coffey C, Posterino M, Carlin JB, Wolfe R, Bowes G (1999) A computerised screening instrument for adolescent depression: population-based validation and application to a two-phase case-control study. Soc Psychiatry Psychiatr Epidemiol 34(No 3):166-172

Rutter M, Quinton D (1984) Parental psychiatric disorder: effects on children. Psych Med 14(4):853-880

Salzman JP (1996) Primary attachment in female adolescents: association with depression, self-esteem, and maternal identification. Psychiatry 59:20-23

Sleep training and controlled crying, Mumsnet, http://www.mumsnet. $\mathrm{com} / \mathrm{babies} /$ sleep-training-and-controlled-crying. Accessed 6th Sept 2012

Thompson R (1994) Emotion regulation: a theme in search of definition in the development of emotion regulation: biological and behavioral considerations. Monogr Soc Res Child Dev 59(2/3):25-52

White I, Royston P, Wood AM (2011) Multiple imputation using chained equations: issues and guidance for practice. Stat Med 30:377-399 